\title{
CONGENITAL HYPOTHYROIDISM IN PRETERM INFANTS: EXPERIENCE IN A SINGLE TERTIARY CENTER IN SOUTHERN THAILAND
}

\author{
1Somchit Jaruratanasirikul, M.D., ${ }^{2}$ Hutcha Sriplung, M.D. \\ 1Department of Pediatrics, ${ }^{2}$ Epidemiology Unit, Faculty of Medicine, \\ Prince of Songkla University, Hat Yai, Songkhla, 90110 Thailand.
}

Background and aims: Preterm infants are at high risk to develop congenital hypothyroidism $(\mathrm{CH})$ due to the immaturity of the hypothalamic-pituitary-thyroid axis, loss of iodine supply from the mother, and preterm health problems. In our institute, Songklanagarind Hospital, thyroid screening in preterm infants was implemented in 2010 . We aimed to study the etiologies of $\mathrm{CH}$ in preterm infants admitted during 2010-2013.

Methods: The medical records of preterm infants who were diagnosed as $\mathrm{CH}$ as defined by TSH level at the time of thyroid screening $>10 \mathrm{mU} / \mathrm{L}$ or free $\mathrm{T} 4$ level $<1.00 \mathrm{ng} / \mathrm{dL}$ were retrospectively reviewed.

\begin{abstract}
Results: There were 1,966 preterm infants. Of these, 52 cases $(2.78 \%)$ were diagnosed with $\mathrm{CH}$. The average TSH levels at the first screening (day 7-8) was $20.35 \mathrm{mU} / \mathrm{L}$ and the confirmatory test (day 16-20) was $13.98 \mathrm{mU} / \mathrm{L}$. After thyroxine discontinuation for 8-10 weeks at 3 years of age, there were 12 patients $(23.1 \%)$ who had TSH levels $>10 \mathrm{mU} / \mathrm{L}$ and were diagnosed as permanent $\mathrm{CH}$. Thyroid scintigraphy revealed ectopic thyroid in 2 patients (16.7\%), thyroid hypoplasia in 1 patient (8.3\%), normal thyroid in 7 patients (58.3\%) and enlarged thyroid in 2 patients (16.7\%). The 40 preterm infants who had normal thyroid function after thyroxine discontinuation for 2 years were diagnosed as transient $\mathrm{CH}$.
\end{abstract}

Table 1. Demographic data in all patients $(N=52)$

\begin{tabular}{|c|c|c|c|c|}
\hline Characteristic & $N=52$ & $\begin{array}{c}\text { Media } \\
\mathrm{n}\end{array}$ & Mean \pm SD & Range \\
\hline Male/Female & $23 / 29$ & - & - & \\
\hline Gestational age, weeks & & 32 & $32.0 \pm 2.53$ & $27-36$ \\
\hline Birth weight, gm & & 1440 & $1449 \pm 477$ & $520-2740$ \\
\hline \multicolumn{5}{|l|}{ Associated disorders, N (\%) } \\
\hline - Sepsis & $23(44.2)$ & & & \\
\hline - Respiratory distress syndrome & $26(50)$ & & & \\
\hline - Ventilator-associated pneumonia & $7(13.5)$ & & & \\
\hline - Necrotizing enterocolitis & $14(26.9)$ & & & \\
\hline - Patent ductus arteriosus & $10(19.2)$ & & & \\
\hline \multicolumn{5}{|l|}{ Initial investigations } \\
\hline Age at first TSH screening, days & & 8 & $8.73 \pm 3.88$ & $3-14$ \\
\hline TSH level, $\mathrm{mU} / \mathrm{L}$ & & 20.35 & $20.85 \pm 12.19$ & $7.72-100$ \\
\hline Age at second screening, days & & 18 & $17.55 \pm 9.33$ & $9-33$ \\
\hline Free $\mathrm{T}_{4}$ level, ng/dL & & 1.39 & $1.45 \pm 0.31$ & $0.89-2.22$ \\
\hline TSH level, mU/L & & 13.98 & $15.22 \pm 5.14$ & 7.38-36.08 \\
\hline Initial dosage of thyroxine, $\mu \mathrm{g} / \mathrm{kg} / \mathrm{d}$ & & 16 & $14.4 \pm 2.65$ & $10-50$ \\
\hline \multicolumn{5}{|l|}{ At 3 years of age } \\
\hline $\begin{array}{l}\text { Duration of thyroxine treatment, } \\
\text { months }\end{array}$ & & 35.1 & $33.0 \pm 5.9$ & $19.6-42.7$ \\
\hline $\begin{array}{l}\text { Interval from thyroxine } \\
\text { discontinuation and thyroid function } \\
\text { test done, weeks }\end{array}$ & & 7.4 & $7.7 \pm 34.60$ & $4-22.8$ \\
\hline Free $\mathrm{T}_{4}$ level, ng/dL & & 1.36 & $1.37 \pm 0.18$ & $1.05-1.83$ \\
\hline TSH level, mU/L & & 5.75 & $6.55 \pm 4.13$ & $0.51-19.25$ \\
\hline Children with TSH >10 mU/L & $12(23.1)$ & - & - & - \\
\hline Age At thyroid scan done, years & $\mathrm{N}=12$ & 3.41 & $3.21 \pm 0.61$ & $2.22-3.84$ \\
\hline Final diagnosis, $\mathrm{n}(\%)$ & & - & - & \\
\hline - $\quad$ Transient hypothyroidism & $40(76.9)$ & - & - & \\
\hline - $\quad$ Persistent mild hypothyroidism & $11(21.2)$ & - & - & \\
\hline - $\quad$ Persistent overt hypothyroidism & $1(1.9)$ & - & - & \\
\hline
\end{tabular}

Table 2. Details of the thyroid function test in 12 preterm infants with permanent congenital hypothyrodism

\begin{tabular}{|c|c|c|c|c|c|c|c|c|c|c|c|c|c|c|}
\hline \multirow[t]{3}{*}{ No } & \multirow{3}{*}{$\begin{array}{l}\text { Se } \\
x\end{array}$} & \multirow{3}{*}{$\begin{array}{l}\text { GA } \\
\text { (wk) }\end{array}$} & \multirow{3}{*}{$\begin{array}{l}\text { BW } \\
\text { (g) }\end{array}$} & \multirow{2}{*}{\multicolumn{2}{|c|}{$\begin{array}{c}\text { First } \\
\text { Screening }\end{array}$}} & \multirow{2}{*}{\multicolumn{2}{|c|}{$\begin{array}{l}\text { Second } \\
\text { screening }\end{array}$}} & \multicolumn{6}{|c|}{ After thyroxine discontinuation } & \multirow[t]{3}{*}{ Thyroid scan } \\
\hline & & & & & & & & \multicolumn{2}{|c|}{ 6-8 weeks } & \multicolumn{2}{|c|}{6 months } & \multicolumn{2}{|c|}{12 months } & \\
\hline & & & & $\mathrm{FT}_{4}$ & TSH & $\mathrm{FT}_{4}$ & TSH & $\mathrm{FT}_{4}$ & TSH & $\mathrm{FT}_{4}$ & $\begin{array}{l}\text { TS } \\
\mathrm{H}\end{array}$ & $\mathrm{FT}_{4}$ & TSH & \\
\hline 1 & $\mathrm{~F}$ & 32 & 2100 & 0.98 & $>100$ & 0.40 & $>100$ & 1.00 & 44.20 & - & - & - & - & Ectopic \\
\hline 2 & M & 36 & 1250 & 1.01 & 40.0 & 0.90 & 20.20 & 1.53 & 19.25 & - & - & - & - & Normal \\
\hline 3 & $\mathrm{~F}$ & 31 & 1730 & 1.20 & 36.0 & 1.48 & 12.6 & 1.20 & 16.50 & - & - & . & - & Ectopic \\
\hline 4 & $\mathrm{~F}$ & 31 & 1460 & 1.10 & 20.3 & 1.44 & 20.3 & 1.36 & 7.20 & 1.22 & 8.85 & 1.37 & 10.4 & Enlarged \\
\hline 5 & $\mathrm{~F}$ & 31 & 1555 & 1.34 & 24.5 & 1.34 & 36.0 & 1.45 & 11.90 & - & - & - & - & Normal \\
\hline 6 & $\mathrm{~F}$ & 35 & 1575 & ND & ND & 1.00 & 17.8 & 1.45 & 10.50 & - & - & - & - & Normal \\
\hline 7 & M & 34 & 1650 & ND & ND & 1.29 & 12.0 & 1.46 & 10.59 & - & - & - & - & Normal \\
\hline 8 & M & 30 & 1060 & ND & ND & 1.42 & 17.9 & 1.15 & 15.10 & - & - & - & - & Normal \\
\hline 9 & M & 35 & 1300 & 1.25 & 12.2 & 1.90 & 13.9 & 1.32 & 5.75 & 1.33 & 15.7 & - & - & Normal \\
\hline 10 & M & 34 & 2740 & 0.78 & 7.78 & 1.01 & 17.3 & 1.17 & 8.48 & 1.10 & 9.98 & 1.16 & 10.2 & Enlarged \\
\hline 11 & M & 29 & 760 & 0.85 & 24.2 & 1.30 & 23.0 & 1.21 & 12.10 & - & - & - & - & Hypoplasia \\
\hline 12 & $\mathrm{~F}$ & 30 & 1062 & - & - & 1.06 & 14.7 & 1.07 & 11.21 & - & - & & - & Normal \\
\hline
\end{tabular}

Table 3. Characteristics of preterm infants with the final diagnosis of transient and mild permanent congenital hypothyrodism

\begin{tabular}{|l|c|c|c|}
\hline & $\begin{array}{c}\text { Transient } \mathrm{CH} \\
(\mathrm{N}=40)\end{array}$ & $\begin{array}{c}\text { Mild } \mathrm{CH} \\
(\mathrm{N}=11)\end{array}$ & P value \\
\hline Initial investigations & & & \\
\hline Age at TSH screening, days & $9.2 \pm 3.1$ & $8.3 \pm 4.7$ & 0.78 \\
\hline TSH level, mU/L & $15.31 \pm 11.61$ & $25.47 \pm 11.52$ & 0.17 \\
\hline Age at confirmation, days & $19.0 \pm 10.7$ & $17.6 \pm 8.9$ & 0.98 \\
\hline Free $\mathrm{T}_{4}$ level, $\mathrm{ng} / \mathrm{dL}$ & $1.49 \pm 0.33$ & $1.27 \pm 0.18$ & 0.10 \\
\hline TSH level, $\mathrm{mU} / \mathrm{L}$ & $14.40 \pm 4.07$ & $18.21 \pm 7.41$ & 0.11 \\
\hline At 3 years of age & & & \\
\hline FT 4 after 6-8 wks discontinuation, $\mathrm{ng} / \mathrm{dL}$ & $1.38 \pm 0.21$ & $1.37 \pm 0.13$ & 0.95 \\
\hline TSH level after 6-8 wks discontinuation, & $4.61 \pm 1.74$ & $11.15 \pm 4.62$ & $<0.001$ \\
\hline
\end{tabular}

Conclusion: $\mathrm{CH}$ is common in preterm infants: $23 \%$ permanent and $77 \%$ transient $\mathrm{CH}$. Thyroid screening is crucial for early detection and treatment of patients with permanent $\mathrm{CH}$ to prevent longterm neurodevelopmental complications of untreated $\mathrm{CH}$. 